\title{
Aerodynamic Modelling and Analysis of a Novel Mechanism for Chord and Camber Morphing Wing
}

\author{
Harun Levent Şahin ${ }^{1,}{ }^{*}$, Bora Orçun Çakır ${ }^{2}$, and Yavuz Yaman ${ }^{3}$ \\ ${ }^{1}$ Graduate Research Assistant, Department of Aerospace Engineering, METU, Republic of Turkey \\ ${ }^{2}$ Undergraduate Student, Department of Aerospace Engineering, METU, Republic of Turkey \\ ${ }^{3}$ Professor, Department of Aerospace Engineering, METU, Republic of Turkey
}

\begin{abstract}
This paper presents the aerodynamic modelling and analysis of surfaces created by a novel deployable mechanism, which is composed of a four-bar linkage and a scissor-structural mechanism (SSM) which contains several scissor-like elements (SLEs). With the help of that mechanism, which is located inside the trailing portion of wing section, continuous adjustment of the airfoil is possible. In order to highlight the advantageous aerodynamic characteristics of newly created airfoil geometries via proposed SSM, several aerodynamic analyses have been performed. The flow characteristics used for the analyses are determined by the flight envelope of an intended generic UAV. Since the maximum speed range of the sample aircraft is well below Mach 0.3, incompressible flow assumption is made throughout the solutions and conservation laws of Reynolds Transport Theorem are employed.
\end{abstract}

\section{Introduction}

From the Wright brothers, man has gone through several structural changes to increase the efficiency of aircraft. Much of these changes have been considered for aircraft wings, on which the principal control elements of aircraft are located. Most of modern aircrafts use conventional control surfaces such as flaps, ailerons, or slats, which allow the aircrafts to fly at a range of flight conditions; however, their performance are not optimal since such a design of control surfaces does not provide a smooth transition of camber in the chord-wise direction [1]. This phenomenon causes a sudden change in the pressure distribution at the hinge line of the control surfaces, and it is usually associated with a drag penalty and the possibility of separation $[2,3]$.

Aircraft morphing, especially "wing morphing", provide the possibility of obtaining an adaptive wing structure which offer radical shape changes to produce optimum performance over an aircraft's nominal operational envelope [4] even expand its operating envelope [5].

There are three generally accepted major groups for morphing aircraft concepts: planform alternation, airfoil adjustment and out-of-plane transformation [6]. Airplanes

* Corresponding author: hlsahin@metu.edu.tr 
generally adjust the airfoil camber by deflecting the conventional control surfaces. Since the trailing edge bears less aerodynamic moment than the leading edge, it is a lot easier to drive and control [7].

In this paper, the aerodynamic behaviors of a novel morphing wing trailing edge structure with various configurations of airfoil shapes are investigated. The aerodynamic analyses of airfoil configurations are performed in 2D by XFOIL, which predicts the pressure distribution over the airfoil which can be integrated to calculate the lift and drag coefficients. The wings have been assumed to have the required leading edge morphing as well in order to have the necessary airfoil shapes.

\section{Design of a scissor-structural mechanism for the morphing of trailing edge of an aircraft wing}

Especially in civil aviation, most of aircrafts have trailing edges with a honeycomb sandwich structure which are actuated by concentrated hydraulic servo mechanisms. Although such designs have the advantage in terms of stiffness and a large \& fast-respond driving force, from a morphing wing standpoint, such designs have a discontinuity at joint position, insufficient in-plane deformability, and a large driving system weight [7]. In order to overcome those problems, a "scissor-structural mechanism" (SSM) is proposed. Scissorstructural mechanisms have the characteristic of both a mechanism and a structure; therefore, they are one type of "structural mechanism" [8].

In this paper, in order to morph the camber line and chord length of an aircraft wing, a SSM is designed to fit into the trailing part of the aircraft wing. For verification of the results, those wing sections are taken as of 4-digit NACA airfoils, which are studied extensively in [9].

Considering the baseline airfoil as NACA 4412 type, a SSM is designed to morph the trailing edge part of the wing section into NACA 8412 airfoil. In designs, three different total number of SLEs $(N=8,10,12)$ are used. Perturbation of geometric properties of SLEs gives several results. The best SSMs, which have the least mean structural errors, are also investigated whether they satisfy the other airfoils (i.e. NACA 2412, NACA 6412). In Table 1, a summary of mean structural errors are given:

Table 1. Mean structural errors for various SSMs with different number of SLEs.

\begin{tabular}{l||c|c|c} 
& $\boldsymbol{N}=\mathbf{8}$ & $\boldsymbol{N}=\mathbf{1 0}$ & $\boldsymbol{N}=\mathbf{1 2}$ \\
\hline \hline NACA 2412 & 0.0016 & 0.0015 & 0.0019 \\
\hline NACA 6412 & 0.0006 & 0.0008 & 0.0008 \\
\hline NACA 8412 & 0.0012 & 0.0017 & 0.0022
\end{tabular}

In Fig. 1, a SSM with $N=8$ SLEs at its initial position is shown. When the anchor link is rotated $\phi=19^{\circ}$ clockwise, the designed SSM will satisfy the NACA 8412 geometry with $0.12 \%$ mean structural error. 


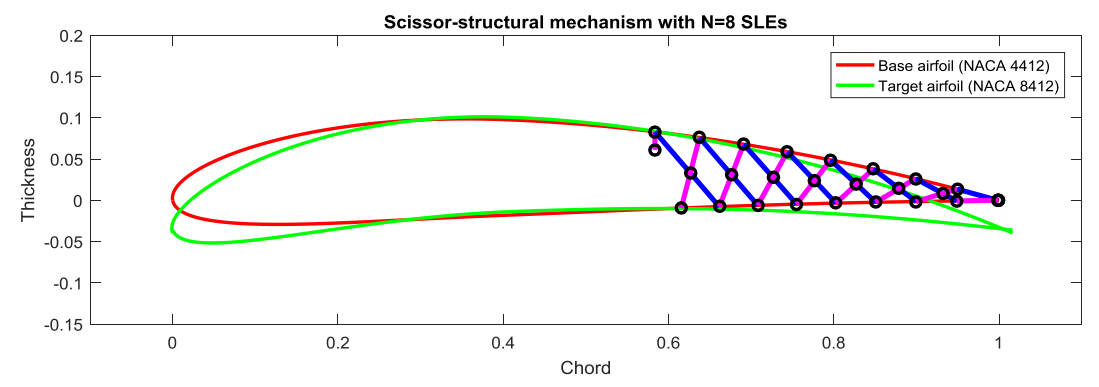

Fig. 1. Scissor-structural mechanism with $\boldsymbol{N}=\mathbf{8}$ SLEs at its initial position.

As a summary, increasing the number of SLEs does not affect the mean structural error but increases the stiffness of the structure and smooths the surface.

\section{Theory}

\subsection{XFOIL: Panel method}

Panel method provides solutions for linear, inviscid, irrotational flows around solid surfaces subjected to subsonic speeds. The solution algorithm employs panels to construct the solid interfaces with vortices and sources inducing a velocity field around the body. Then, the system of equations is solved by corresponding boundary conditions defined over the airfoil matching the number of panels. The corresponding pressure distribution is calculated using the tangential velocity components distributed over the surface, which also leads by integration over the airfoil contour to the computation of lift and drag forces. Nevertheless, owing to the fact that formulation of panel method considers inviscid flow conditions; skin friction component caused by interactions between a solid body and a viscous fluid is not taken into account. Thus, the total drag force calculated with panel method, normally consisting of skin friction and pressure drag components, lacks the skin friction drag component which leads to significant undershoots in terms of drag force and significant overshoots in terms of lift force computations. Furthermore, not only miscalculation of drag force but also absence of boundary layer interactions contributes to the possible inaccuracies of panel method especially for high angle of attack (AoA) values. These effects and their various performance characteristics are well-defined in literature including comparisons with experimental results [10].

In order provide a solution to the problem of lacking viscous contribution, XFOIL is developed with a viscous coupling formulation where the entire viscous solution (boundary layers and wake) is strongly interacted with the incompressible potential flow via the surface transpiration model. This permits proper calculation of limited separation regions. The drag is determined from the wake momentum thickness far downstream. A special treatment is used for a blunt trailing edge, which fairly and accurately accounts for base drag [11].

\subsection{Validation of solver}

Owing to the fact that NACA 4412 airfoil is used as the baseline airfoil for the morphing modifications, the panel method based solver XFOIL is validated over experimental results presented at NACA Report No. 586 [12] and NACA Report No. 646 [13]. Viscous solutions of six different Reynolds numbers attained from XFOIL are compared with the experimental results in terms of pressure coefficient distributions over the chord length and 
variation of lift coefficient with different AoA values. In order to keep the validation process compatible with the solutions, $n_{\text {panel }}=200$ panels are assigned over the airfoil coordinates focused on leading and trailing edges, and the results are compared considering the uncertainty limits included in the experimental data.

Throughout the solutions performed using XFOIL iteration number is limited to $n_{\text {iter }}=100$ which is accompanied with a root-mean-square (RMS) tolerance of tol= $10^{-4}$ for convergence. Since the panel method provides an inviscid solution for the flow around an airfoil, XFOIL possess an additional viscous coupling to be able to generate viscous solutions and boundary layer interactions over the airfoil surfaces. However, it is well documented in the literature that, for high AoA values, it has a tendency to overshoot the lift coefficient values with reduced effect of boundary layer interactions and separation due to adverse pressure gradient [14]. Hence, both for the validation and the solution processes, AoA values higher than $\alpha=15^{\circ}$ and lower than $\alpha=-5^{\circ}$ were avoided. Consequently, according to the limitations and parameters selected, solutions obtained with Reynolds number of $R e=330000$ revealed best agreement with the experimental data.

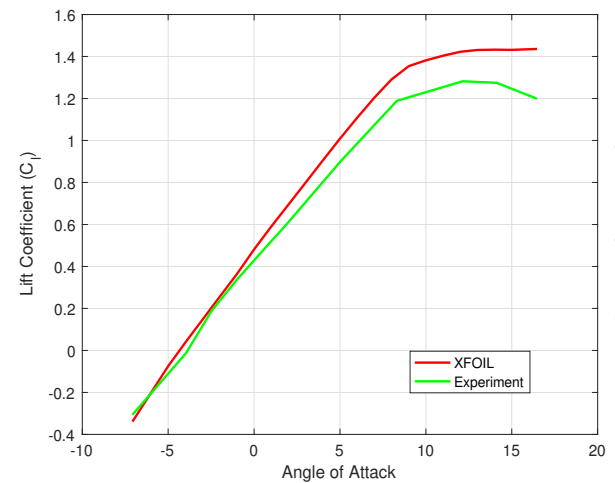

(a)

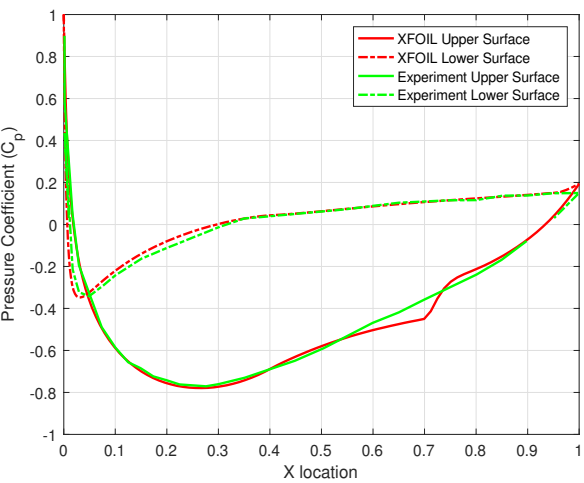

(b)

Fig. 2. Comparison of (a) lift coefficient $\left(\boldsymbol{C}_{\boldsymbol{l}}\right)$ vs. angle of attack $(\boldsymbol{\alpha})$, (b) pressure coefficient distribution $\left(\boldsymbol{C}_{\boldsymbol{p}}\right)$ obtained by XFOIL and experimental data for NACA 4412 type airfoil.

\section{Aerodynamic performance of scissor-structural mechanisms for chord and camber morphing wing}

Aerodynamic analyses have been conducted for three different total number of SLEs $(N=8,10,12)$. Table 2 gives a summary of RMS errors of pressure coefficient $\left(C_{p}\right)$ distributions calculated regarding the original NACA airfoils and the surfaces generated by proposed SSMs:

Table 2. RMS errors of pressure distributions for various SSMs with different number of SLEs.

\begin{tabular}{c||c|c|c} 
& $\boldsymbol{N}=\mathbf{8}$ & $\boldsymbol{N}=\mathbf{1 0}$ & $\boldsymbol{N}=\mathbf{1 2}$ \\
\hline \hline NACA 2412 & 0.2348 & 0.0575 & 0.1574 \\
\hline NACA 6412 & 0.3165 & 0.3047 & 0.3043 \\
\hline NACA 8412 & 0.2337 & 0.1434 & 0.1663
\end{tabular}


Fig. 3 summarizes the results obtained from the XFOIL for SSM with $N=8$ SLEs at three different poses (NACA 2412, NACA 6412, NACA 8412). As seen from the figure, the results nearly overlap with the original NACA airfoils; however, at the point, where the anchor link of the mechanism is located, pressure coefficient fluctuates due to the sharp decrease in panel number.

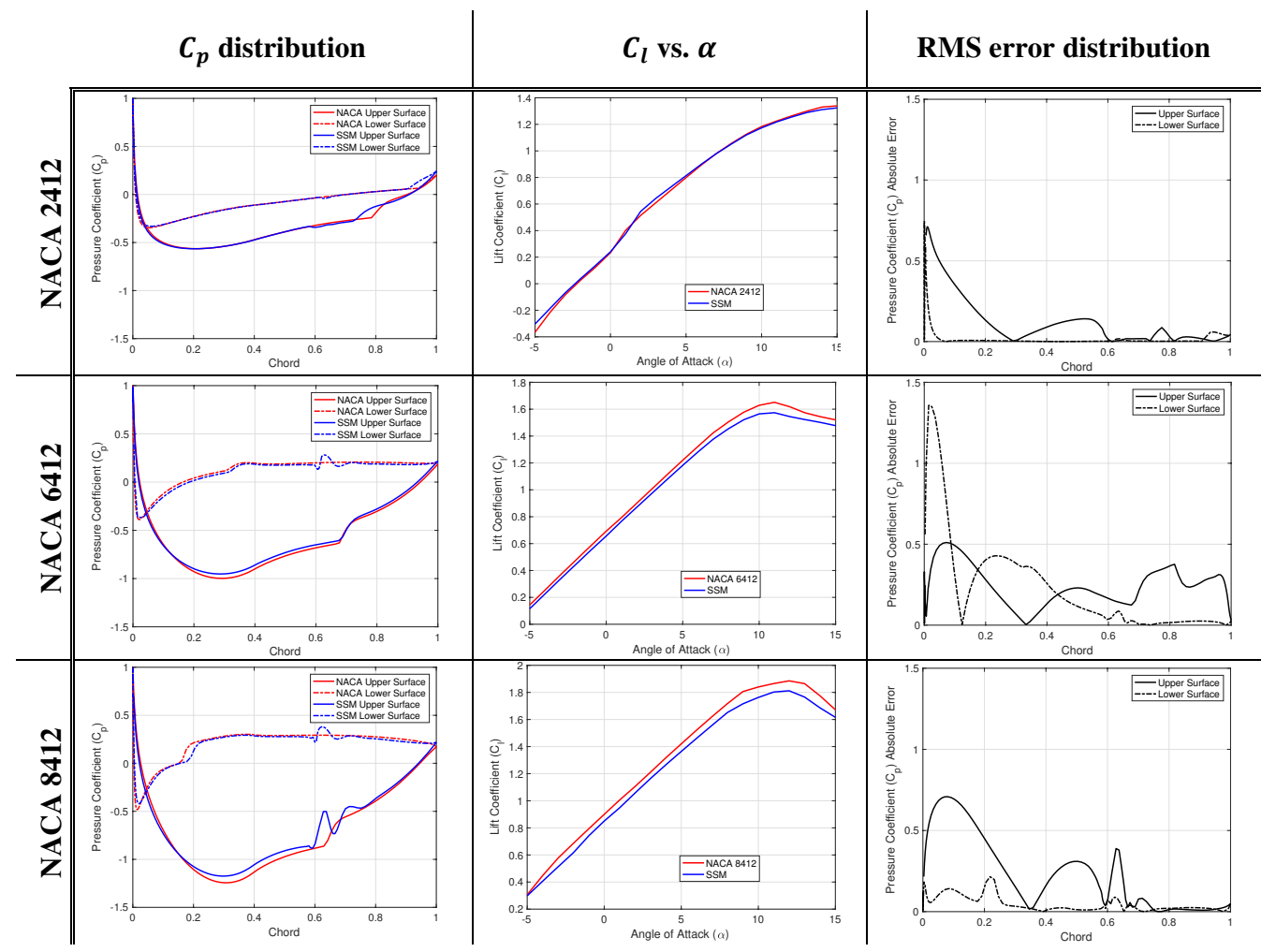

Fig. 3. Comparison of aerodynamic behavior of proposed SSM with $\boldsymbol{N}=\mathbf{8}$ SLEs at three different poses with original NACA airfoils.

Fig. 4 and Fig. 5 summarizes the results for SSMs with $N=10$ and $N=12$ SLEs at three different poses respectively. It is clearly seen that, the fluctuation occurred at the beginning of the SSM melt away as one increase the used total number of SLEs. Although the mean structural error does not vary, aerodynamics performance of SSMs with higher total number of SLEs are better. 


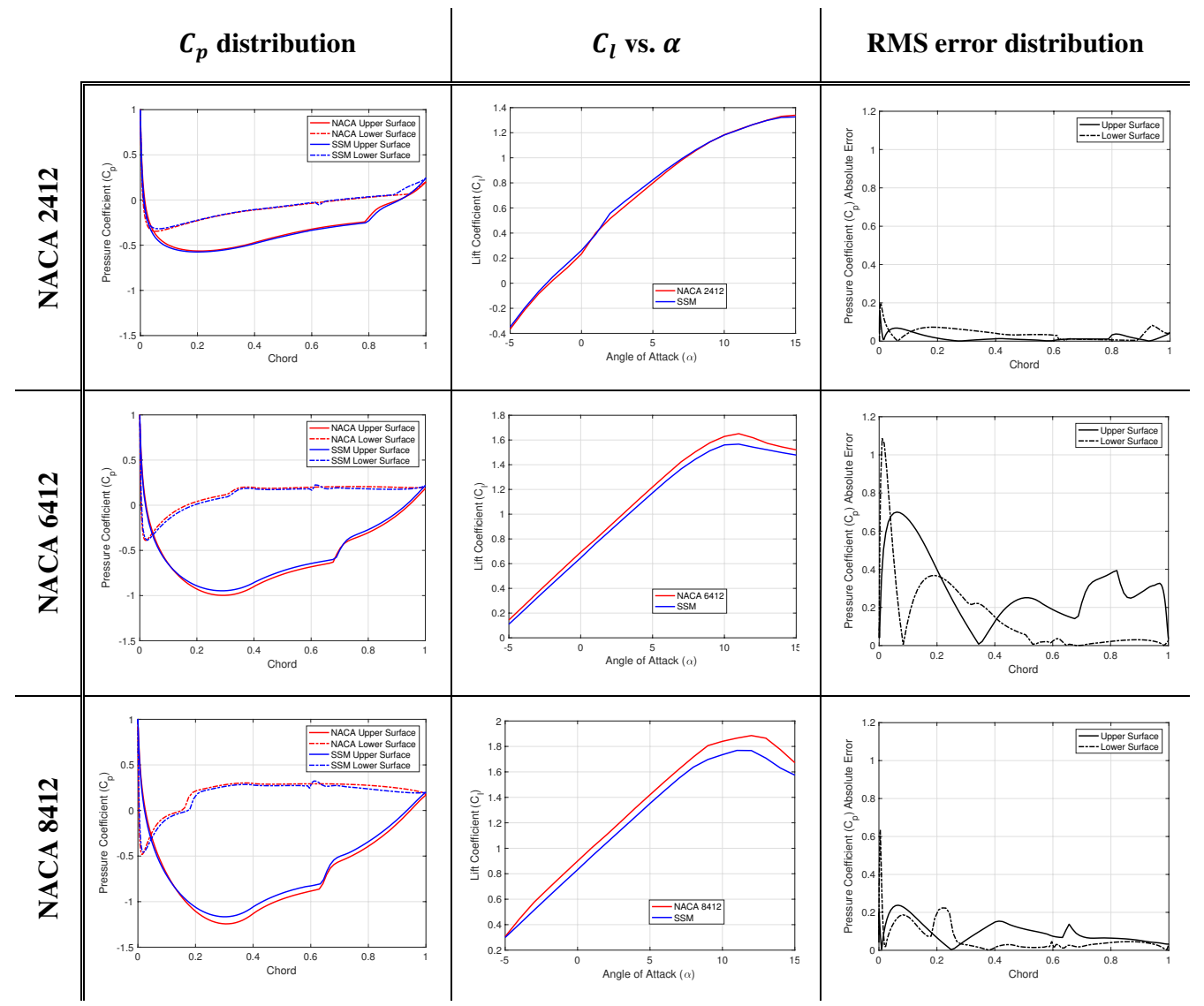

Fig. 4. Comparison of aerodynamic behavior of proposed SSM with $\boldsymbol{N}=10$ SLEs at three different poses with original NACA airfoils. 


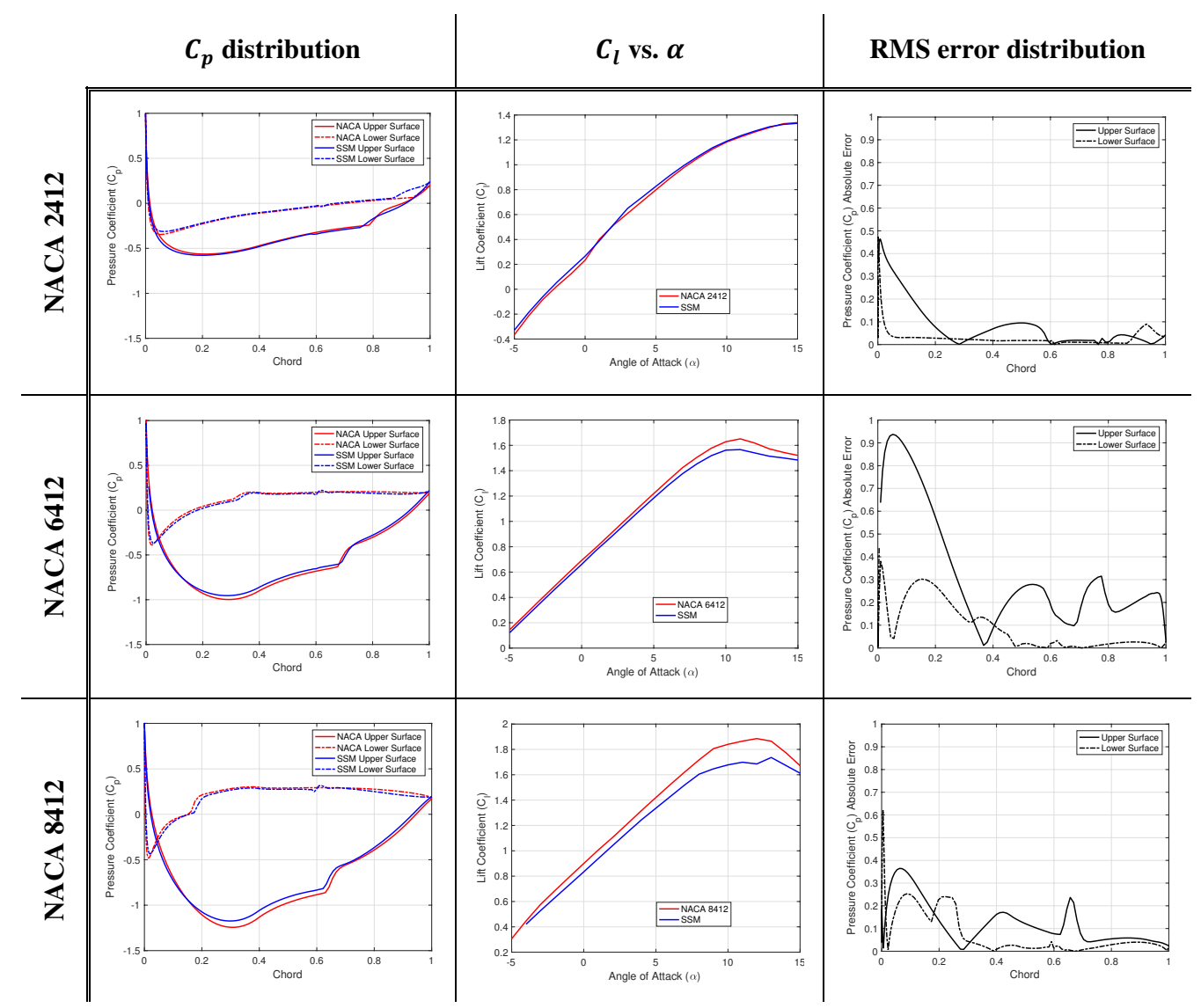

Fig. 5. Comparison of aerodynamic behavior of proposed SSM with $\boldsymbol{N}=\mathbf{1 2}$ SLEs at three different poses with original NACA airfoils.

\section{Conclusion}

In this article, an ongoing research about morphing of the trailing edge of an aircraft wing is presented. Assuming that aircraft wing skins are flexible enough to generate the desired motion of the internal mechanism, a scissor-structural mechanism for the morphing of trailing edge of an aircraft wing is designed. Since designed scissor-structural mechanism afford the target profiles with little mean structural errors, in order to ensure their aerodynamic performance, the profiles obtained from proposed mechanisms are modelled and analyzed aerodynamically. The obtained results are compared with the original NACA airfoils.

As seen from the results, designed scissor-structural mechanisms for each case satisfy the target airfoil profiles successfully with little lift penalty. Since the proposed SSMs have the capability to obtain high camber rates, they can also generate higher lifts than NACA airfoils. 


\section{References}

1 S. Barbarino, O. Bilgen, R. M. Ajaj, M. I. Friswell, and D. J. Inman, J. Intell. Mater. Syst. Struct. 22 (2011) 823-877.

2 J. H. S. Fincham, R. M. Ajaj, and M. I. Friswell, 14th AIAA Aviat. Technol. Integr. Oper. Conf. (Atlanta, 2014).

3 B. Woods, J. H. S. Fincham, J. H. S. Fincham, and M. I. Friswell, 14th AIAA Aviat. Technol. Integr. Oper. Conf. (Atlanta, 2014).

4 J. H. S. Fincham and M. I. Friswell, Aerosp. Sci. Technol. 43, (2015) 245-255.

$5 \quad$ K. R. Olympio and F. Gandhi, J. Intell. Mater. Syst. Struct. 21 (2010) 1719-1735.

6 A. Y. N. Sofla, S. A. Meguid, K. T. Tan, and W. K. Yeo, Mater. Des. 31 (2010) 1284-1292.

$7 \quad$ P. Zhang, L. Zhou, W. Cheng, and T. Qiu, J. Aircr. 52 (2015) 452-461.

8 Y. Akgün, C. J. Gantes, W. Sobek, K. Korkmaz, and K. Kalochairetis, Eng. Struct. 33 (2011) 1365-1376.

9 H. L. Şahin, and Y. Yaman, 5th International Conference of Engineering Against Failures (Chios Island, 2018).

10 D. Bertetta, S. Brizzolara, S. Gaggero, M. Viviani, and L. Savio, Sustainable Maritime Transportation and Exploitation of Sea Resources - Proceedings of the 14th International Congress of the International Maritime Association of the Mediterranean (Genova, 2012).

11 M. Drela, XFOIL: Proceedings of the Conference Notre Dame (Indiana, 1989).

12 T. J. R. Hughes, (Prentice-Hall, 1987).

13 C. G. Davila, P. P. Camanho, and C. A. Rose, J. Compos. Mater. 39 (2005) 323345.

14 H. Liu, IOP Conf. Series: Materials Science and Engineering 326 (2018) 012016. 